\title{
Outage Probability for Directional Beamforming in High Density Wireless Networks
}

\author{
Ayman T. Abusabah ${ }^{\dagger \ddagger}$ and Rodolfo Oliveira ${ }^{\dagger \ddagger}$ \\ †Departamento de Engenharia Electrotécnica, Faculdade de Ciências e Tecnologia, FCT, \\ Universidade Nova de Lisboa, 2829-516 Caparica, Portugal \\ $\ddagger_{\text {IT, Instituto de Telecomunicações, Portugal }}$ \\ emails: a.sabah@campus.fct.unl.pt,rado@fct.unl.pt
}

\begin{abstract}
In this paper, we derive the outage probability in directional millimeter-wave communications, capturing the effect of beamforming. In the proposed scenario, multiple transmitters are spatially distributed according to a spatial Poisson Point Process, which can cause interference to a pair of transmitter/receiver communicating nodes. The analysis considers a general distancebased path loss with Rayleigh and Rician fading channels and a sectored antenna model. Assuming that nodes are uniformly distributed over a circular or annular area centered at the receiver, we derive the outage probability due to the aggregate interference caused by multiple transmitters. The outage probability is based on the Signal-to-interference-plus-noise ratio (SINR) derived for the transmitter/receiver pair. Several simulations confirm the effectiveness of the derived results for different wireless channels and beamwidth values, highlighting the effect of directional communications on the outage probability.

Keywords-Directional Beamforming, Interference, Perfor-
\end{abstract} mance Analysis.

\section{INTRODUCTION}

The recent advances in millimeter-wave (mmWave) wireless communication systems are now capable of providing high data rates up to several gigabits per second in portions of the spectrum never used before. The high frequencies at mmWave bands limit the communication range. Moreover, mmWave systems' efficiency is much higher for line-of-sight (LoS) channels and take advantage of recent techniques such as multi-input and multi-output (MIMO). Regarding the main techniques adopted in the physical layer of mmWave systems, we highlight the adoption of spatial multiplexing capable of subdividing the transmitted signal into multiple spatial streams simultaneously transmitted over the same channel but from different antennas. Additionally, the existing mmWave systems take advantage of classical array processing techniques capable of directing the beam pattern by phasing multiple antenna elements, aka beamforming (BF). BF has interesting properties for short-range communication systems, because they may achieve higher frequency spatial reuse depending on how the spatial activation of the directional beams are scheduled [1].

Nowadays, the role of mmWave communication systems schemes in short-range wireless communications is very important. These include several initiatives at the $60 \mathrm{GHz}$ band, such as next-generation personal area networks and wireless local area networks [2]. Motivated by the adoption of mmWave communication systems in short-range networks exhibiting high spatial density of transmitters (TXs) per area unit, in this work we evaluate the performance of a mmWave communication link when exposed to the interference caused by multiple TXs closely located to the link's receiver (RX).

The outage probability of beamforming systems was studied in several works considering intrinsic aspects such as imperfect beamforming [3] and co-channel interference [4]. These works do not account for the interference caused by multiple TXs but only due to impairments and other propagation phenomena. The characterization of the interference caused by multiple mmWave beamforming TXs has been addressed in [5] considering that the interferers are located according to a spatial binomial point process. A spatial Poisson Point Process (PPP) was adopted in [6] to derive the outage probability caused by beamforming TXs that access the channel from multiple random locations. The work in [7] considered the outage analysis for random device-to-device networks underlying mmWave cellular networks. Moreover, the work in [8] explored the possibility of base station co-operation in the downlink of mmWave heterogeneous networks to reduce the outage caused by high dense deployment, shadowing, and blockage at mmWave band.

Differently from [6]-[8], in this work all TXs adopt a beamforming sectored antenna model with different side lobe gains according to the beamwidth and the boresight angle, contributing to a more realistic antenna model. We assume an operational scenario where multiple interferers are spatially located according to a spatial PPP. Then we derive a simple model to characterize the outage probability of the mmWave communication link. The model takes into account the interference caused by multiple TXs considering the spatial distribution of the nodes, different radio propagation channels, and directional beamforming. To characterize the outage probability we derive the distributions of the aggregate interference and the received power at the $\mathrm{RX}$, which are used to derive a closed expression for the outage probability. The probability is validated using simulation results that confirm the accuracy of the proposed model.

Section II introduces the system model. Section III derives the outage probability of the mmWave link. The accuracy of the proposed model is evaluated in Section IV. Finally, Section $\mathrm{V}$ concludes the paper.

Regarding the notation adopted in the paper, $f_{X}($.$) is$ 
adopted to represent the Probability Density Function (PDF) of a Random Variable (RV) $X . \mathbb{P}[X=x]$ and $\mathrm{E}[X]$ represent the probability and the expectation, respectively. $\varphi_{X}$ is the Characteristic Function (CF) of $X . \Gamma(x)$ and $\Gamma(p, x)$ are the Gamma function and the incomplete gamma function, respectively. ${ }_{2} \mathrm{~F}_{1}(a, b ; c ; z)$ denotes the Gauss Hypergeometric function.

\section{System Model And Assumptions}

\section{A. Network Model}

We consider a scenario where nodes are distributed according to a homogeneous $\mathrm{PPP}^{1}$ with density $\lambda$ over an annular region with an inner radius, $R_{\mathcal{I}}$, and an outer radius, $R_{\mathcal{O}}$, i.e., $R_{\mathcal{I}} \leq r \leq R_{\mathcal{O}}$. For the particular case of $R_{\mathcal{I}}=0$ a circular region is considered. Without loss of generality, we condition on a reference RX at the origin, which according to Slivnyak's theory in stochastic geometry [9], yields to a homogeneous PPP with the corresponding density $\lambda$. At a given time slot, we assume that the reference RX is connected with one TX and other TXs may cause interference to the reference RX. We also consider a single-hop transmission only, with the same transmission power for all nodes.

\section{B. Gain Pattern and Beamforming Modeling}

All nodes are assumed to be equipped with antenna arrays for performing directional beamforming. We adopt a sectorized antenna model as depicted in Fig. 1, to represent the gain pattern $G_{T}$ and $G_{R}$ at the TX and RX, respectively, as follows

$$
G_{T, R}(\theta)=\left\{\begin{array}{ll}
G^{\max }, & \text { if }|\theta| \leq \omega / 2 \\
G^{\min }, & \text { if }|\theta| \geq \omega / 2
\end{array},\right.
$$

where $G^{\max }$ and $G^{\text {min }}$ are the gains of main and side lobes, respectively, defined by a beamwidth $\omega \in(0,2 \pi)$ and the boresight angle direction $\theta \in[-\pi, \pi)$. Without loss of generality, we assume that all nodes are on the same horizontal plane, i.e., no variation in beam pattern over the elevation angle, and we work with the normalized 2-D pattern. While the antenna modeling might look quite simplified, it was adopted in [10] for establishing innovative MAC policies and has been considered in other works [11], [12].
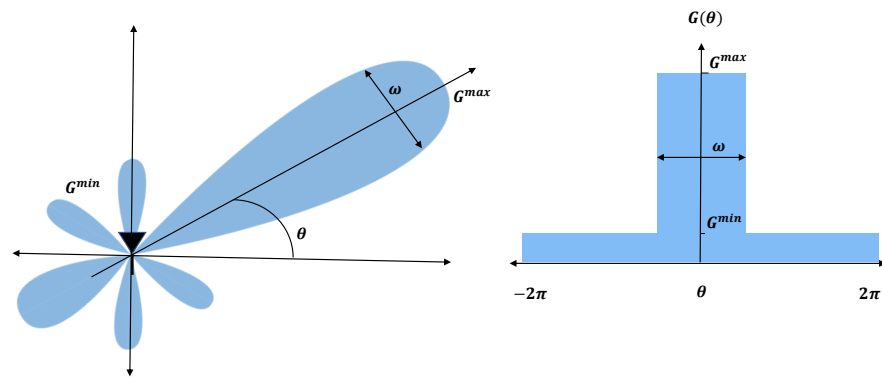

Fig. 1. Beamforming sectored antenna model.

\footnotetext{
${ }^{1}$ The PPP has convenient mathematical properties and has been adopted as a mathematical model for seemingly random processes in the discipline.
}

The RX at the origin and its corresponding TX are assumed to steer their gain patterns toward each other. Assuming perfect estimation of angles of arrival and no alignment errors, the directivity gain of the intended link is $G_{\mathcal{I}}=G_{T}^{\max } G_{R}^{\max }$. The beam orientation of interferers is assumed to be uniformly distributed in $[-\pi, \pi)$. Since the output of (1) is $G^{\max }$ or $G^{\text {min }}$ over $[-\pi, \pi)$, the resulting gain distributions of an $i$-th interfering node observed at the RX, $G_{i, \mathcal{I}}(\theta)$, or observed at the TX, $G_{\mathcal{I}, i}$, are discrete and their probability density function $(\mathrm{PDF})$ is given by

$$
f_{G_{i, \mathcal{I}}}(g)=f_{G_{\mathcal{I}, i}}(g)=p \delta\left(g-G^{\max }\right)+\bar{p} \delta\left(g-G^{\min }\right),
$$

where $p=\omega / 2 \pi$ is the main lobe hit rate and $\bar{p}=1-p$ is the main lobe miss rate between interferers and the RX.

\section{Communication Model}

We consider a large-scale, distance-based path loss and small-scale fading for modeling the wireless channel. Therefore, the received power caused by an $i$-th node at the RX is given by

$$
P_{i}=P_{t} H_{i} G_{i, \mathcal{I}} G_{\mathcal{I}, i} r_{i}^{-\alpha},
$$

where $P_{t}$ is the same transmitted power across all nodes. We consider both Rayleigh and Rician small-scale fading channels in which $H_{i}$ is drawn from a Gamma distribution as will be shown later. Moreover, $G_{i, \mathcal{I}}$ and $G_{\mathcal{I}, i}$ represent the gain of the $i$-th TX and the gain of the RX, respectively. $\alpha>2$ is the large-scale path loss coefficient, and $r_{i}$ is the distance between an $i$-th TX and the reference RX. To avoid $P_{i}>P_{t}$ in the near field, we consider that $R_{\mathcal{I}} \geq 1$, [13].

The outage occurs when the received signal-to-interferenceplus-noise ratio (SINR) at the reference RX is below a defined SINR threshold, $\gamma_{o}>0$, which can be formulated as follows

$$
P_{\text {out }}=\mathbb{P}\left\{\gamma \leq \gamma_{o}\right\}=\int_{0}^{\gamma_{o}} f_{\gamma}(z) d z,
$$

where $P_{\text {out }}$ is the outage probability, $\gamma$ and $f_{\gamma}(z)$ represent the SINR at the reference RX and its corresponding PDF, respectively. Based on (3), $\gamma$ can be defined as

$$
\gamma=\frac{P_{\mathcal{I}}}{Y}
$$

where $P_{\mathcal{I}}=P_{t} G_{\mathcal{I}} H_{i} r_{i}^{-\alpha}$ is the intended received power, i.e., the power received at the reference $\mathrm{RX}$ and transmitted by a corresponding TX. Moreover, $Y=I_{o}+\eta$, where $\eta$ represents the background noise power, while $I_{o}$ is the aggregate interference power seen at the reference RX and written as follows

$$
I_{o}=\sum_{i=1}^{N} P_{i}, i \in \Pi,
$$

where $N$ is a RV characterized by a homogeneous PPP that represents the number of active TXs over the region given by the area $A=\pi\left(R_{\mathcal{O}}^{2}-R_{\mathcal{I}}^{2}\right)$. Our goal is to compute $P_{\text {out }}$ which requires characterizing $\gamma$ as explicitly shown in (4). In the next section, we describe the required steps for characterizing $\gamma$ and consequently obtaining $P_{\text {out }}$. 


\section{Computation of Outage Probability}

To compute $P_{\text {out }}$ in (4), it is required to derive $f_{\gamma(z)}$. The distribution of $\gamma$ is merely the ratio distribution between $P_{\mathcal{I}}$ and $Y$. Therefore, we start the analysis by characterizing the RVs $P_{\mathcal{I}}$ and then $Y$.

\section{A. Characterization of $P_{\mathcal{I}}$}

The distribution of $P_{\mathcal{I}}=P_{t} G_{\mathcal{I}} H_{i} r_{i}^{-\alpha}$ is a product between the constants $\left(P_{t} G_{\mathcal{I}}\right)$ and the RVs $H_{i}$ and $r_{i}^{-\alpha}$. Both Rayleigh and Rician small-scale fading channels are considered. The Rayleigh channel can describe the stochastic fading when there is no LoS signal. When the channel is a Rayleigh distributed, $H_{i}$ can be drawn from an exponential distribution with mean $\frac{1}{\mu}$, therefore, it can be expressed by a Gamma distribution with the shape parameter, $k=1$, and the scale parameter, $\theta=\frac{1}{\mu}$, as follows

$$
H_{i} \sim \operatorname{Exp}(\mu) \sim \operatorname{Gamma}(k, \theta) .
$$

On the other hand, the Rician fading channel is parameterized through $K$ and $\Omega$ where $K$ represents the quotient between the power in the LoS component and the power in the other nonLoS components, and $\Omega$ represents the total power from both components. Then, the received signal amplitude is Rician distributed with parameters $\nu^{2}=\frac{K \Omega}{1+K}$ and $\sigma^{2}=\frac{\Omega}{2(1+K)}$. $K_{d B}=10 \log _{10}(K)$ is the decibels representation of $K$. By definition, if $X \sim \operatorname{Rice}(\nu, \sigma)$, then $\left(\frac{X}{\sigma}\right)^{2}$ follows a non-central Chi-squared distribution with 2 degrees of freedom and noncentrality parameter $\left(\frac{\nu}{\sigma}\right)^{2}$. Consequently, moment matching can then be used to obtain a simplified Gamma approximation for $H_{i}$ as follows

$$
H_{i} \sim \operatorname{Gamma}(k, \theta),
$$

where $k$ and $\theta$ are the shape and scale parameters, respectively, given by [14]

$$
k=\frac{\left(\nu^{2}+2 \sigma^{2}\right)^{2}}{4 \sigma^{2}\left(\nu^{2}+\sigma^{2}\right)}, \theta=\frac{4 \sigma^{2}\left(\nu^{2}+\sigma^{2}\right)}{\left(\nu^{2}+2 \sigma^{2}\right)^{2}} .
$$

By definition, if $X \sim \operatorname{Gamma}(k, \theta)$ and $c>0, c X \sim$ $\operatorname{Gamma}(k, c \theta)$, therefore

$$
H_{\mathcal{I}_{i}} \sim \operatorname{Gamma}\left(k, \theta_{\mathcal{I}}\right)
$$

where $H_{\mathcal{I}_{i}}=P_{t} G_{\mathcal{I}} H_{i}$ and $\theta_{\mathcal{I}}=P_{t} G_{\mathcal{I}} \theta$. Note that, $k$ and $\theta$ are defined as in (7) for the Rayleigh channel and as in (8) for the Rician channel.

Lets assume that $L_{i}=r_{i}^{\alpha}$ is the RV representing the path loss, $P_{\mathcal{I}}$ can be written as

$$
P_{\mathcal{I}}=\frac{H_{\mathcal{I}_{i}}}{L_{i}}
$$

Since the nodes are uniformly distributed over $A=\pi\left(R_{\mathcal{O}}^{2}-\right.$ $R_{\mathcal{I}}^{2}$ ), the probability that an interfering node is positioned at distance $r$ from the $\mathrm{RX}$ is given by the $\mathrm{CDF} F_{R}(r)=$ $\frac{\left(r^{2}-R_{\mathcal{I}}^{2}\right)}{\left(R_{\mathcal{O}}^{2}-R_{\mathcal{I}}^{2}\right)}$, which yields to the PDF $f_{R}(r)=\frac{2 r}{\left(R_{\mathcal{O}}^{2}-R_{\mathcal{I}}^{2}\right)}$. Consequently, the CDF and PDF of $L_{i}$ are respectively stated as follows

$$
F_{L_{i}}(l)=\frac{\left(l^{\frac{2}{\alpha}}-R_{\mathcal{I}}^{2}\right)}{\left(R_{\mathcal{O}}^{2}-R_{\mathcal{I}}^{2}\right)}, \quad \text { for } R_{\mathcal{I}}^{\alpha} \leq l \leq R_{\mathcal{O}}^{\alpha},
$$

$$
f_{L_{i}}(l)=\frac{2 l^{\frac{2}{\alpha}-1}}{\alpha\left(R_{\mathcal{O}}^{2}-R_{\mathcal{I}}^{2}\right)}, \quad \text { for } R_{\mathcal{I}}^{\alpha} \leq l \leq R_{\mathcal{O}}^{\alpha} .
$$

Assuming that the RV $H_{\mathcal{I}_{i}}$ is independent of the RV $L_{i}$, then, the PDF of $P_{\mathcal{I}}$ is simply given by the ratio distribution as follows

$$
f_{P_{\mathcal{I}}}(s)=\int_{-\infty}^{\infty}|l| f_{H_{\mathcal{I}_{i}}}(l s) f_{L_{i}}(l) d l
$$

The integral in (13) can be solved by replacing $f_{L_{i}}(l)$ by (12b) and $f_{H_{\mathcal{I}_{i}}}(l s)$ by (10), yields to

$$
f_{P_{\mathcal{I}}}(s)=\int_{R_{\mathcal{I}}^{\alpha}}^{R_{\mathcal{O}}^{\alpha}}|l| \frac{(l s)^{k-1} e^{\frac{-l s}{\theta_{\mathcal{I}}}}}{\Gamma(k) \theta_{\mathcal{I}}^{k}} \frac{2 l^{\frac{2}{\alpha}-1}}{\alpha\left(R_{\mathcal{O}}^{2}-R_{\mathcal{I}}^{2}\right)} d l .
$$

After solving the integral in (14), the PDF of $P_{\mathcal{I}}$ is written as

$$
\begin{aligned}
f_{P_{\mathcal{I}}}(s)= & \left(\Gamma\left[k+\frac{2}{\alpha}, \frac{R_{\mathcal{I}}^{\alpha} s}{\theta_{\mathcal{I}}}\right]-\Gamma\left[k+\frac{2}{\alpha}, \frac{R_{\mathcal{O}}^{\alpha} s}{\theta_{\mathcal{I}}}\right]\right) \\
& \times \frac{2 s^{-\frac{2+\alpha}{\alpha}} \theta_{\mathcal{I}}^{\frac{2}{\alpha}}}{\alpha\left(R_{\mathcal{O}}^{2}-R_{\mathcal{I}}^{2}\right) \Gamma(k)} .
\end{aligned}
$$

\section{B. Characterization of $P_{i}$}

Since the aggregate interference power, denoted by $I_{o}$, represents the summation of the power generated by each node, we first start our analysis by characterizing the interference power caused by an individual node. According to (3), $P_{i}$ can be seen as a product of $P_{t}$ and the RVs $H_{i}, G_{i}$, and $r_{i}^{-\alpha}$, where $G_{i}=G_{i, \mathcal{I}} G_{\mathcal{I}, i}$ is the directivity gain of an $i$ th interfering link. Similar to III-A, $P_{t} H_{i} \sim \operatorname{Gamma}\left(k, \theta_{o}\right)$, where $\theta_{o}=P_{t} \theta$. Consequently, the PDF of $S_{i}=\frac{P_{t} H_{i}}{L_{i}}$ can be concluded as follows

$$
\begin{aligned}
f_{S_{i}}(s)= & \left(\Gamma\left[k+\frac{2}{\alpha}, \frac{R_{\mathcal{I}}^{\alpha} s}{\theta_{o}}\right]-\Gamma\left[k+\frac{2}{\alpha}, \frac{R_{\mathcal{O}}^{\alpha} s}{\theta_{o}}\right]\right) \\
& \times \frac{2 s^{-\frac{2+\alpha}{\alpha}} \theta_{o}^{\frac{2}{\alpha}}}{\alpha\left(R_{\mathcal{O}}^{2}-R_{\mathcal{I}}^{2}\right) \Gamma(k)},
\end{aligned}
$$

The RV $P_{i}$ represents a product between the RVs $S_{i}$ and $G_{i}$. Actually, $G_{i}$ can be seen as a product of two random gains, $G_{i, \mathcal{I}}$ and $G_{\mathcal{I}, i}$, where each follows the PDF in (2). Intuitively, the possible set of outputs for $G_{i}$ are $\left\{G^{\max ^{2}}, G^{\text {min }^{2}}\right.$, $\left.G_{\max } G_{\min }\right\}$ with probabilities $\left\{p^{2}, \bar{p}^{2}, 2 p \bar{p}\right\}$, respectively. Therefore, the PDF of $G_{i}$ can be expressed as follows

$$
\begin{aligned}
f_{G_{i}}(g)= & p^{2} \delta\left(g-G^{\text {max }^{2}}\right)+\bar{p}^{2} \delta\left(g-G^{\text {min }^{2}}\right) \\
& +2 p \bar{p} \delta\left(g-G^{\text {max }} G^{\text {min }}\right),
\end{aligned}
$$

where $p^{2}$ represents the probability that the TX main lobe and RX main lobe hit each other, $\bar{p}^{2}$ represents the probability that the TX main lobe and RX main lobe miss each other, and $2 p \bar{p}$ represents the probability that one hits and the other misses.

The PDF of $P_{i}$ can then be found by the classical product distribution as follows

$$
f_{P_{i}}(x)=\int_{-\infty}^{\infty} \frac{1}{|g|} f_{G_{i}}(g) f_{S_{i}}(x / g) d g .
$$


This integral can be solved by replacing $f_{G_{i}}(g)$ and $f_{S_{i}}(x / g)$ by (17) and (16), respectively, obtaining

$$
\begin{aligned}
& f_{P_{i}}(x)=\frac{p^{2}}{G^{\max ^{2}}} f_{S_{i}}\left(\frac{x}{G^{m a x^{2}}}\right)+\frac{\bar{p}^{2}}{G^{\min ^{2}}} f_{S_{i}}\left(\frac{x}{G^{\min ^{2}}}\right) \\
& +\frac{2 p \bar{p}}{G^{\min } G^{\max }} f_{S_{i}}\left(\frac{x}{G^{\min } G^{\max }}\right) .
\end{aligned}
$$

Consequently, the MGF of $P_{i}$, defined as $\Phi_{P_{i}}(s)=E\left[e^{s P_{i}}\right]$ is represented in (20) where $\varrho_{1}(x)={ }_{2} \mathrm{~F}_{1}\left(k, \frac{-2}{\alpha}, \frac{-2+\alpha}{\alpha}, x\right)$.

\section{Characterization of $Y$}

According to (6), the RV $I_{O}$ represents the summation of $N$ independent RVs $\left(I_{o}=P_{1}+P_{2}+\ldots+P_{N}\right)$. As mentioned before, $N$ follows a homogeneous PPP distribution where

$$
P[N=n]=\frac{(\lambda|A|)^{n}}{n !} e^{-\lambda|A|}
$$

Since $P_{i}$ are independent, then for a specific number of active interferers $n$, we can write

$$
\Phi_{I_{o} \mid n}(s)=\Phi_{P_{1}}(s) \times \Phi_{P_{2}}(s) \cdots \times \Phi_{P_{n}}(s)=\left(\Phi_{P_{i}}(s)\right)^{n} .
$$

Using the law of total probability, the PDF of the aggregate interference $I_{o}$ can be expressed as

$$
f_{I_{o}}(j)=\sum_{n=0}^{\infty} f_{I_{o}}(j \mid N=n) P[N=n] .
$$

Thus, the MFG of the aggregate interference, $\Phi_{I_{o}}(s)$, can be written as

$$
\begin{aligned}
\Phi_{I_{o}}(s) & =\mathrm{E}\left[e^{s I_{o}}\right]=\sum_{n=0}^{\infty} P[N=n] \int_{-\infty}^{\infty} e^{s j} f_{I_{o}}(j \mid N=n) \\
& =\sum_{n=0}^{\infty} P[N=n] \Phi_{I_{o} \mid n}(s)=\sum_{n=0}^{\infty} \frac{\left(\lambda|A| \Phi_{P_{i}}(s)\right)^{n}}{n !} e^{-\lambda|A|}
\end{aligned}
$$

As $e^{x}=\sum_{n=0}^{\infty}\left(x^{n}\right) / n !$, (24) can be expressed as follows

$$
\Phi_{I_{o}}(s)=e^{\lambda|A|\left(\Phi_{P_{i}}(s)-1\right)}
$$

We can obtain the $k$-th moment of $I_{O}$ from (25), i.e., $\mathrm{E}\left[I_{o}^{k}\right]=\left.\frac{d^{k}}{d s^{k}} \Phi_{I_{o}}(s)\right|_{s=0}$. For the approximation of $Y=I_{o}+\eta$, the moments can then be matched with the respective moments of a given distribution. Empirical data was used in a maximum log-likelihood estimation process to determine the parameters of the known distributions, where the Gamma distribution achieved the highest accuracy in representing the aggregate interference. This observation is confirmed by [15], where it is shown that aggregate interference due to large-scale and smallscale fading can be approximated by a Gamma distribution.

Therefore, the PDF of $Y$ can be written as follows

$$
f_{Y}(y)=\frac{y^{k_{e q}-1} e^{\frac{-y}{\theta_{e q}}}}{\theta_{e q}^{k_{e q}} \Gamma\left(k_{e q}\right)}, \quad \text { for } y>0,
$$

where the shape and scale parameters, $k_{e q}$ and $\theta_{e q}$, respectively, are given by

$$
k_{e q}=\frac{\mathrm{E}[Y]^{2}}{\mathrm{E}\left[Y^{2}\right]-\mathrm{E}[Y]^{2}}, \quad \theta_{e q}=\frac{\mathrm{E}\left[Y^{2}\right]-\mathrm{E}[Y]^{2}}{\mathrm{E}[Y]},
$$

with $\mathrm{E}[Y]=E\left[I_{o}\right]+\eta$ and $\mathrm{E}\left[Y^{2}\right]=E\left[I_{o}^{2}\right]+2 \eta E\left[I_{o}\right]+\eta^{2}$, where $E\left[I_{o}\right]$ and $E\left[I_{o}^{2}\right]$ are given in (28a) and (28b), respectively.

\section{Computation of $P_{\text {out }}$}

Given that $P_{\mathcal{I}}$ and $Y$ are independent RVs, then, $P_{\text {out }}$ can be formulated as follows

$$
P_{\text {out }}=\int_{0}^{\gamma_{o}} \int_{0}^{\infty} y f_{P_{\mathcal{I}}}(y z) f_{Y}(y) d y d z .
$$

Solving (29) yields to a closed-form expression for $P_{\text {out }}$ given by (30), where where $\varrho_{2}(x)={ }_{2} \mathrm{~F}_{1}\left(k_{e q}, k+k_{e q}, 1+k_{e q}, x\right)$, and $\varrho_{3}(x)={ }_{2} \mathrm{~F}_{1}\left(k+k_{e q}, k_{e q}-\frac{2}{\alpha}, 1+k_{e q}-\frac{2}{\alpha}, x\right)$.

\section{Performance Analysis}

This section presents numerical results based on the proposed model and provides some interpretations. The numerical results obtained with the model are compared with the results obtained through Monte Carlo simulation results $\left(10^{6}\right.$ realizations of the stochastic process were run) to evaluate the accuracy of the derived outage probability in directional communication. The network and channel parameters are listed in Table I, unless otherwise specified.

\section{TABLE I}

NETWORK AND CHANNEL PARAMETERS.

\begin{tabular}{|c|c||c|c|}
\hline Symbol & Value & Symbol & Value \\
\hline$P_{t}$ & $1 \mathrm{~mW}$ & $R_{\mathcal{I}}, R_{\mathcal{O}}$ & $1 \mathrm{~m}, 5 \mathrm{~m}$ \\
\hline$\mu$ & 2 & $\lambda$ & $\{10,20,30,50\}$ nodes $/ \mathrm{m}^{2}$ \\
\hline$K_{d B}$ & 0 & $G^{\text {max }}$ & 1 \\
\hline$\Omega$ & $1 \mathrm{~mW}$ & $G^{\text {min }}$ & $\{0.05,0.10\}$ \\
\hline$\alpha$ & 4 & $\omega$ & $\left\{45^{\circ}, 60^{\circ}\right\}$ \\
\hline$\eta$ & $10^{-12} \mathrm{~W}$ & $\gamma_{o}$ & $\{-30,-25,-20,-15,-10,-5\}$ \\
\hline
\end{tabular}

First, The outage probability, $P_{\text {out }}$, is plotted versus different SINR thresholds, $\gamma_{o}$, considering various densities for the nodes, as shown in Fig. 2. The results clearly indicate higher outage values when adopting higher nodes density. This can

$$
\begin{aligned}
\Phi_{P_{i}}(s)= & \frac{1}{R_{\mathcal{I}}^{2}-R_{\mathcal{O}}^{2}}\left(p^{2} R_{\mathcal{I}}^{2} \varrho_{1}\left(G^{\text {max } 2} R_{\mathcal{I}}^{-\alpha} s \theta_{o}\right)+\bar{p}^{2} R_{\mathcal{I}}^{2} \varrho_{1}\left(G^{m i n}{ }^{2} R_{\mathcal{I}}^{-\alpha} s \theta_{o}\right)+2 p \bar{p} R_{\mathcal{I}}^{2} \varrho_{1}\left(G^{\text {max }} G^{\text {min }} R_{\mathcal{I}}^{-\alpha} s \theta_{o}\right)-p^{2} R_{\mathcal{O}}^{2} \varrho_{1}\left(G^{\text {max }} R_{\mathcal{O}}^{-\alpha} s \theta_{o}\right)\right. \\
& \left.-\bar{p}^{2} R_{\mathcal{O}}^{2} \varrho_{1}\left(G^{\text {min } 2} R_{\mathcal{O}}^{-\alpha} s \theta_{o}\right)-2 p \bar{p} R_{\mathcal{O}}^{2} \varrho_{1}\left(G^{\text {max }} G^{\text {min }} R_{\mathcal{O}}^{-\alpha} s \theta_{o}\right)\right)
\end{aligned}
$$




$$
\begin{gathered}
\mathrm{E}\left[I_{o}\right]=\frac{2 A e^{A\left((p+\bar{p})^{2}-1\right) \lambda} k\left(p G^{\text {max }}+\bar{p} G^{\text {min }}\right)^{2} R_{\mathcal{I}}^{-\alpha} R_{\mathcal{O}}^{-\alpha}\left(R_{\mathcal{I}}^{\alpha} R_{\mathcal{O}}^{2}-R_{\mathcal{I}}^{2} R_{\mathcal{O}}^{\alpha}\right) \theta_{o} \lambda}{\left(R_{\mathcal{I}}^{2}-R_{\mathcal{O}}^{2}\right)(-2+\alpha)} \\
\mathrm{E}\left[I_{o}^{2}\right]=\frac{A e^{A\left((p+\bar{p})^{2}-1\right) \lambda} k\left(R_{\mathcal{I}} R_{\mathcal{O}}\right)^{-2 \alpha} \theta_{o}^{2} \lambda}{\left(R_{\mathcal{I}}^{2}-R_{\mathcal{O}}^{2}\right)^{2}}\left(\frac{(1+k)\left(p G^{m a x}+\bar{p} G^{m i n}\right)^{2}\left(R_{\mathcal{I}}^{2}-R_{\mathcal{O}}^{2}\right)\left(R_{\mathcal{I}}^{2 \alpha} R_{\mathcal{O}}^{2}-R_{\mathcal{I}}^{2} R_{\mathcal{O}}^{2 \alpha}\right)}{-1+\alpha}\right. \\
\left.+\frac{4 A k\left(p G^{\text {max }}+\bar{p} G^{\text {min }}\right)^{4}\left(R_{\mathcal{I}}^{\alpha} R_{\mathcal{O}}^{2}-R_{\mathcal{I}}^{2} R_{\mathcal{O}}^{\alpha}\right)^{2} \lambda}{(-2+\alpha)^{2}}\right)
\end{gathered}
$$

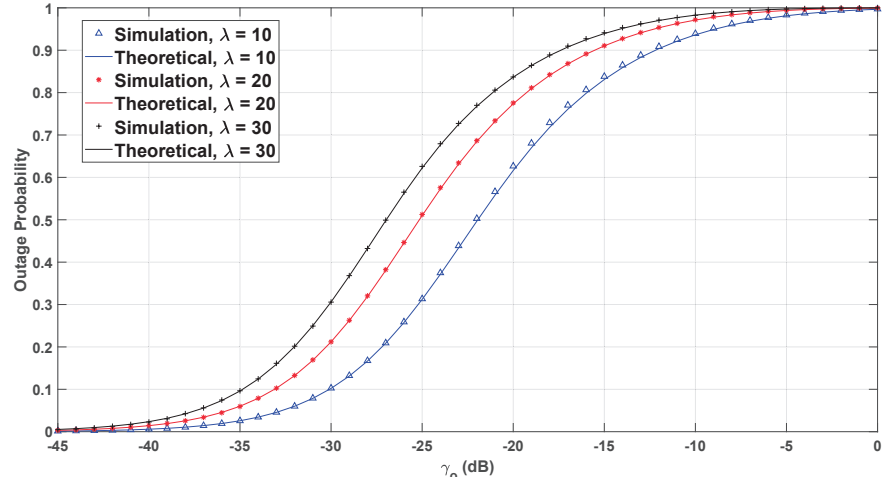

Fig. 2. Outage probability $P_{\text {out }}$ considering different density of the nodes for a Rician fading channel with parameters $K_{d B}=0, \nu=0.707, \sigma=0.5$, $G^{\text {min }}=0.1$, and $\omega=45^{\circ}$.

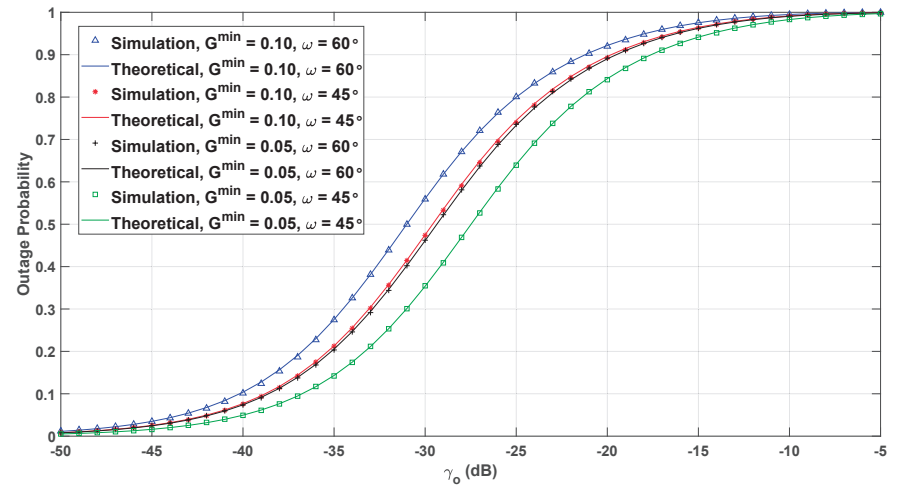

Fig. 3. Outage probability $P_{\text {out }}$ considering a Rayleigh fading channel for $\mu=2$ and $\lambda=50$. be attributed to the higher aggregate interference caused by more TXs.

The outage probability is plotted again versus different SINR thresholds for a Rayleigh fading channel as illustrated in Fig. 3. To demonstrate the effect of directional beamforming, different gain levels of the beam side lobes and different beamwidths are considered. The "simulation" curves were obtained through Monte Carlo simulation while "theoretical" curves were obtained with the computation of the $P_{\text {out }}$ given in (30). The similarity comparison between the simulated data and the theoretical derivations indicates the high accuracy of the proposed approximation. Besides, the results reflect the effect of the side lobes on the outage probability where the results show that the probability of outage increases when considering higher side lobes gain, i.e., $G^{\text {min }}=0.10$ over $G^{\text {min }}=0.05$, as depicted in Fig. 3. On the other hand, the results indicate that the outage probability decreases when adopting small $\omega$ values (narrower beams), i.e., $\omega=45^{\circ}$ over $\omega=60^{\circ}$, due to the concentration of higher power in specific and smaller spatial regions.

Fig. 4 shows the outage probability when considering a Rician fading channel. Once again the same conclusions can be taken with regards to the increase in the outage probability due to the increase in the side lobes gain and the decrease in the outage probability as the beams become narrower.

\section{Conclusions}

This work derives the outage probability by characterizing the SINR considering the effect of beamforming when the nodes are distributed according to a homogeneous PPP. In addition to the large-scale path loss, the proposed model considers two types of small-scale fading channels, Rayleigh and Rician. The proposed approach and derivations were validated through simulations, by considering different channel

$$
\begin{aligned}
P_{\text {out }}= & \frac{\left(R_{\mathcal{I}} R_{\mathcal{O}}\right)^{-k_{e q} \alpha}\left(\gamma_{o} \theta_{e q}\right)^{-k_{e q}} \theta_{\mathcal{I}}^{k_{e q}}}{\left(R_{\mathcal{I}}^{2}-R_{\mathcal{O}}^{2}\right)}\left(R_{\mathcal{I}}^{2} R_{\mathcal{O}}^{k_{e q} \alpha}\left(\frac{\gamma_{o} R_{\mathcal{I}}^{\alpha} \theta_{e q}}{\theta_{\mathcal{I}}}\right)^{k_{e q}}-R_{\mathcal{I}}^{k_{e q} \alpha} R_{\mathcal{O}}^{2}\left(\frac{\gamma_{o} R_{\mathcal{O}}^{\alpha} \theta_{e q}}{\theta_{\mathcal{I}}}\right)^{k_{e q}}+\frac{1}{\left(-2+k_{e q} \alpha\right) \Gamma[k] \Gamma\left[1+k_{e q}\right]} \Gamma\left[k+k_{e q}\right]\right. \\
& \left(-R_{\mathcal{I}}^{2} R_{\mathcal{O}}^{k_{e q} \alpha}\left(-2+k_{e q} \alpha\right) \varrho_{2}\left(-\frac{R_{\mathcal{I}}^{-\alpha} \theta_{\mathcal{I}}}{\gamma_{o} \theta_{e q}}\right)+k_{e q} R_{\mathcal{I}}^{2} R_{\mathcal{O}}^{k_{e q} \alpha} \alpha \varrho_{3}\left(-\frac{R_{\mathcal{I}}^{-\alpha} \theta_{\mathcal{I}}}{\gamma_{o} \theta_{e q}}\right)+R_{\mathcal{I}}^{k_{e q} \alpha} R_{\mathcal{O}}^{2}\left(\left(-2+k_{e q} \alpha\right) \varrho_{2}\left(-\frac{R_{\mathcal{O}}^{-\alpha} \theta_{\mathcal{I}}}{\gamma_{o} \theta_{e q}}\right)\right.\right. \\
& \left.\left.\left.-k_{e q} \alpha \varrho_{2}\left(-\frac{R_{\mathcal{O}}^{-\alpha} \theta_{\mathcal{I}}}{\gamma_{o} \theta_{e q}}\right)\right)\right)\right)
\end{aligned}
$$




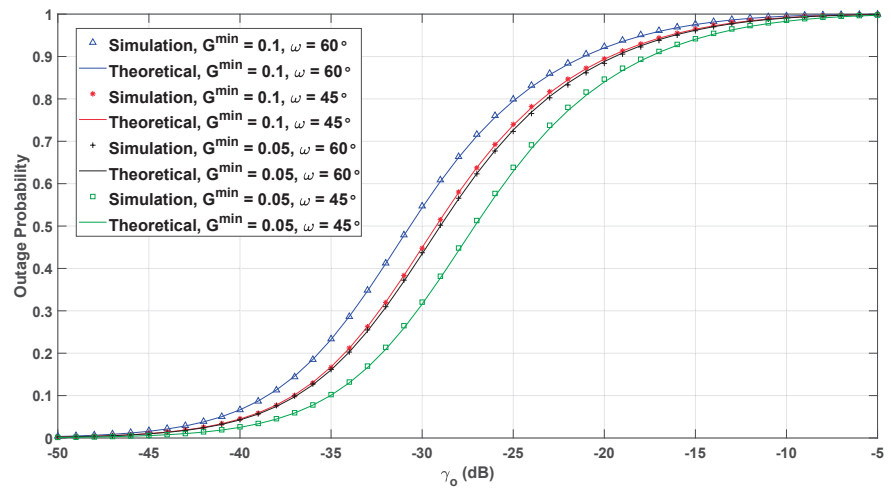

Fig. 4. Outage probability $P_{\text {out }}$ considering a Rician fading channel with parameters $K_{d B}=0, \nu=0.707, \sigma=0.5$, and $\lambda=50$.

and gain pattern parameters, confirming the effectiveness and the accuracy of the proposed approximation.

Finally, we highlight that the adopted beamforming model (two lobes approximation) does not represent the actual antenna array. The assumption of multi lobes will be studied in a complementary way to the study we present, being a subject to be addressed in future works.

\section{ACKNOWLEDGEMENTS}

This work has received funding from the European Union's Horizon 2020 research and innovation programme under the Marie Skłodowska-Curie ETN TeamUp5G, grant agreement No. 813391, and partially supported by the projects CoSHARE (LISBOA-01-0145-FEDER0307095 - PTDC/EEITEL/30709/2017), and UIDB/50008/2020.

\section{REFERENCES}

[1] S. Sun, T. S. Rappaport, R. W. Heath, A. Nix, and S. Rangan. Mimo for millimeter-wave wireless communications: beamforming, spatial multiplexing, or both? IEEE Communications Magazine, 52(12):110 $121,2014$.

[2] C. Park and T. S. Rappaport. Short-range wireless communications for next-generation networks: Uwb, $60 \mathrm{ghz}$ millimeter-wave wpan, and zigbee. IEEE Wireless Communications, 14(4):70-78, 2007.

[3] H. Li, Y. . Yao, and J. Yu. Outage probabilities of wireless systems with imperfect beamforming. IEEE Transactions on Vehicular Technology, 55(5):1503-1515, 2006.

[4] L. Yang and M. Alouini. Outage probability of distributed beamforming with co-channel interference. IEEE Communications Letters, 16(3):334 337, 2012.

[5] S. Niknam, B. Natarajan, and R. Barazideh. Interference analysis for finite-area $5 \mathrm{~g}$ mmwave networks considering blockage effect. IEEE Access, 6:23470-23479, 2018.

[6] T. Samarasinghe, H. Inaltekin, and J. S. Evans. On the outage capacity of opportunistic beamforming with random user locations. IEEE Transactions on Communications, 62(8):3015-3026, 2014.

[7] Sachitha Kusaladharma, Zhang Zhang, and Chintha Tellambura. Interference and outage analysis of random $\mathrm{d} 2 \mathrm{~d}$ networks underlaying millimeter-wave cellular networks. IEEE Transactions on Communications, 67(1):778-790, 2019.

[8] D. Maamari, N. Devroye, and D. Tuninetti. Coverage in mmwave cellular networks with base station co-operation. IEEE Transactions on Wireless Communications, 15(4):2981-2994, 2016.

[9] F. Baccelli and B. Blaszczyszyn. Stochastic Geometry and Wireless Networks. Delft, The Netherlands: Now Publishers, Sep.2009.
[10] S. Singh, R. Mudumbai, and U. Madhow. Interference analysis for highly directional 60-ghz mesh networks: The case for rethinking medium access control. IEEE ACM Trans Netw, 19(5):1513-1527, 2011.

[11] M. Di Renzo. Stochastic geometry modeling and analysis of multitier millimeter wave cellular networks. IEEE Trans. Wireless Commun., 14(9):5038-5057, 2015.

[12] J. Wildman and P. H. J. Nardelli and M. Latva-aho and S. Weber. On the Joint Impact of Beamwidth and Orientation Error on Throughput in Directional Wireless Poisson Networks. IEEE Trans. Wireless Commun, 13(12):7072-7085, 2014.

[13] J. Venkataraman, M. Haenggi, and O. Collins. Shot noise models for outage and throughput analyses in wireless ad hoc networks. In MILCOM 2006 - 2006 IEEE Military Communications conference, pages $1-7,2006$.

[14] A. T. Abusabah, L. Irio, R. Oliveira, and D. B. da Costa. Approximate distributions of the residual self-interference power in multi-tap fullduplex systems. IEEE Wireless Commun. Lett., pages 1-1, 2020.

[15] Martin Haenggi and Radha Ganti. Interference in large wireless networks. Foundations and Trends in Networking, 3:127-248, 012009. 